\title{
THE OPTICAL METHODS ON MEASURING SURFACE DEFORMATION AND SURFACE WAVE IN THE THERMAL CAPILLARY CONVECTION
}

\author{
L. Duan Q. Kang \\ National Microgravity Laboratory/CAS; Institute of Mechanics, \\ Chinese Academy of Sciences, Beijing 100080, China
}

Corresponding author L. Duan

\begin{abstract}
An optical diagnostic system consisting of the Michelson interferometer with the image processor has been developed for the study of the kinetics of the thermal capillary convection. The capillary convection, surface deformation, surface wave and the velocity field in a rectangular cavity with different temperature's sidewalls have been investigated by optical interference method and PIV technique. In order to calculate the surface deformation from the interference fringe, Fourier transformation is used to grating analysis. The quantitative results of the surface deformation and surface wave have been calculated from the interference fringe pattern.
\end{abstract}

Key words: Surface deformation, surface wave, convection, PIV, Interferometer

\section{Introduction}

The thermal capillary convection is driven by the asymmetry of the surface tension, which comes from the asymmetry of the surface temperature. It is an important fluid convection phenomenon in many areas, such as crystal growth and film science. All these physical phenomena are very important influencing factors to many physics process. Lucubrating this convection phenomenon could help people control the physical process of many materials growing, and obtain the perfect result. During the thermal capillary convection occurs, the surface deformation and the surface wave exist on the liquid surface, the surface wave is one of the essential characters in the process of flow translating ${ }^{[1]}$. It is important to study them for understanding the mechanism of the thermal capillary convection. By now, studying the surface of the thermal capillary convection is focused on theories ${ }^{[2]}$, but our research work is experimental study. In the present work, the technique of the optical interference has been introduced to the measurement of the fluid mechanics to establish and develop an optical method, which is used to measure the surface deformation of the flow. The optical diagnostic system consisting of the Michelson interferometer increases the measuring sensitivity in comparison with the conventional experimental method in the fluid mechanics, and the image processor has been developed for obtaining the quantitative results. Combining this optical interference method with the particle image velocimetry (PIV) not only observe the surface deformation and surface wave, but also measure the velocity field of the thermal capillary convection. The optical interferometry and the PIV technique have been proved to be good measurement for the study of the fluid flow. The interesting experiment results are given in the present paper.

\section{The experiment model}

Figure 1 shows a schematic diagram of the experimental facility. The horizontal cross-section of the container is rectangular with the cross-section of $42 \mathrm{~mm} \times 26 \mathrm{~mm}$. The two opposite lateral walls are made of transparent K9 glass for flow visualization and PIV applications. The thickness of the K9 glass is $6 \mathrm{~mm}$. Another two opposite lateral walls are made of copperplates whose thickness is also $6 \mathrm{~mm}$. One of the copperplates is heated using the electro-thermal films another one is cooled by the semiconductor cooling sheet and a radiator. The temperature difference between the two copperplates will form, it will be measured by thermocouple, and the high temperature side is controlled by the DC 
power source. The bottom of the cavity is made by adiabatic materials, whose surface can not reflect the light. There is $4 \mathrm{~mm}$ in height of the silicon oil layer in the experimental cavity. In the present experiment, the temperature difference was increased gradually. The flow in the liquid layer will change from steady convection to un-steady convection. PIV technique and optical interference method have been used to measure the velocity, surface deformation and surface wave, in order to analyze the relation and the mechanism between the thermal capillary convection and the surface deformation and the surface wave.

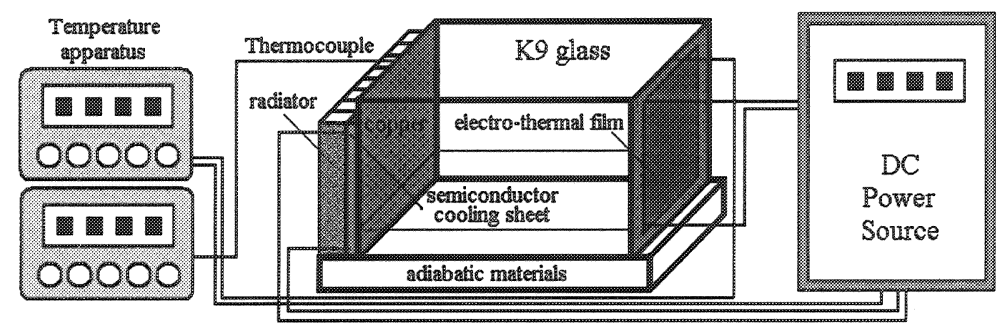

Fig. 1 Schematic diagram of the experiment apparatus

The optical interference method is promising technique without disturbance for measuring mini physics quantity. It is usually used to measure the surface change in solid mechanics, and usually used to measure the temperature, the density and the concentration in the fluid for a long time. But, the surface deformation of the fluid has not been observed using the optical interference method. No disturbing to the measured section is it's most excellent character. In the present experiment, we try to use the Michelson interference method to measure the liquid surface whose measured area is in micron.

The diagram of the optical diagnostic system is shown in Fig. 2. A He-Ne laser beam passes through the lens L1 and L2 to form an expanded parallel light beam, and then the beam is splitted by the splitter Bs into two parallel light beams. One is used as the object beam, and another is used as the reference beam. The object light beam illuminates the liquid surface, and reflects from the liquid surface, then reflects by splitter Bs. The reference light beam is reflected by the mirror M4, and then passes through the splitter Bs. At this time, the object beam and the reference beam meet together and form an interferometric fringe pattern on the ground glass $D$. The fringes carry out the surface deformation of the capillary convection. The image system is composed by the CCD camera and DVR station in the computer. The area measured by the interferometer is $20 \mathrm{~mm} \times 16 \mathrm{~mm}$, which is at the center of the cavity for the reason of horizontal liquid surface.

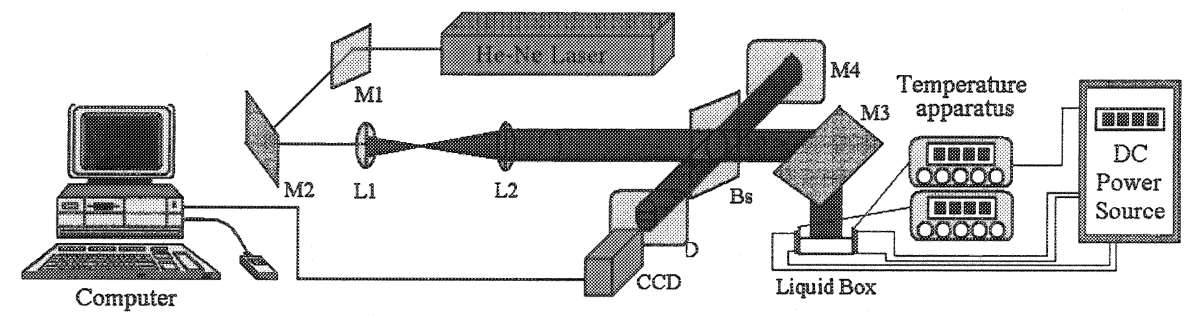

Fig. 2 Optical interference system

In order to calculate the surface deformation from the interference fringe, grating analysis has been used ${ }^{[3-5]}$. We use the fringe at $0^{\circ} \mathrm{C}$ temperature difference as the original grating, and the fringe we want obtain the surface deformation as the metamorphic grating. The phase distribution of the original grating and the metamorphic grating has been calculated from the follows: Fourier transformation is used to transform the two gratings, the cross phase is calculated, and the wrapped phase is unwrapped. 
The phase of the metamorphic grating minus the phase of the original grating, obtains the phase distribution, which introduced from the surface deformation. According to the relationship between the phase $\Phi$ and the displacement $u=\Phi / 2 \pi f$, the surface deformation has been calculated.

According to the upper image processing method, several simulations of fringes calculation have been calculated, which proved that the image processing method is reasonable. Fig. 3(a) is the original fringe pattern, Fig. 3(b) is the metamorphic fringe pattern, the quantitative result of the cylindrical surface has been calculated by Fourier transformation method shown as in Fig. 3(c). The similar calculation is shown as in Fig. 4.

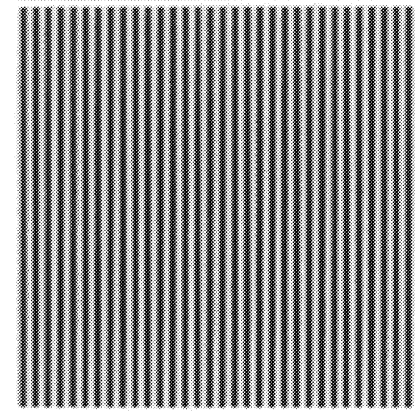

(a)

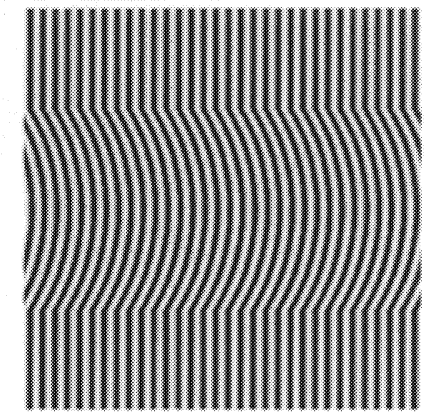

(b)

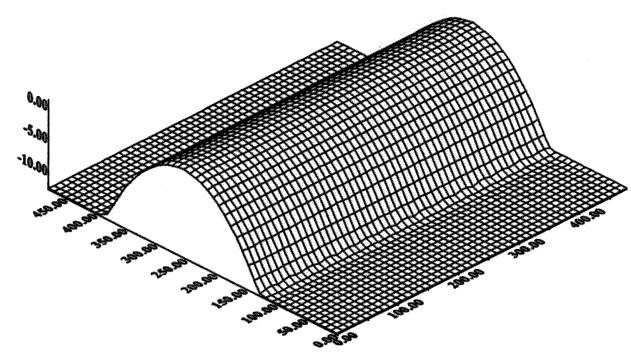

(c)

Fig. 3 One of the simulations of fringes calculation

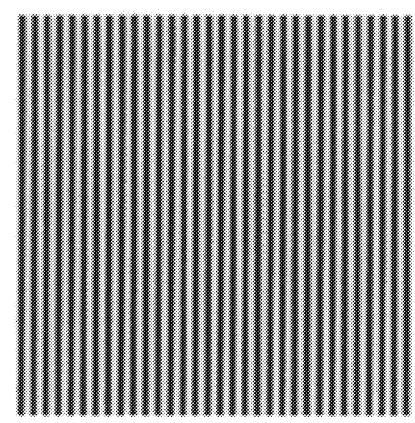

(a)

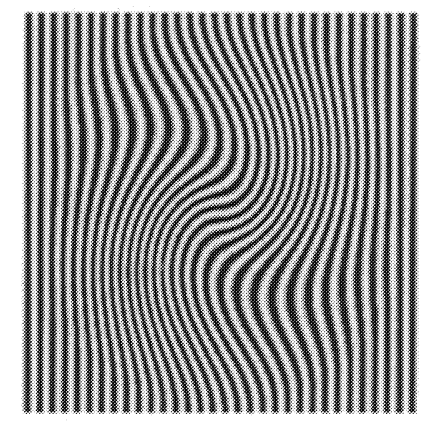

(b)

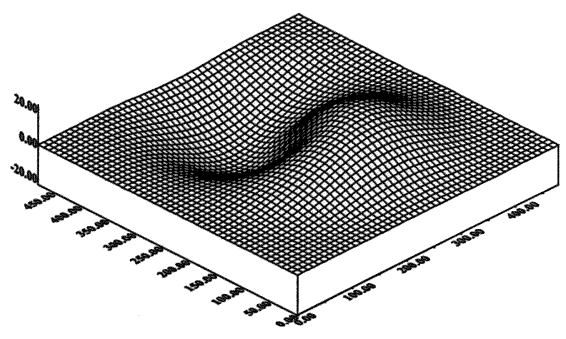

(c)

Fig. 4 Another one of the simulations of fringes calculation

In order to study the relationship between the surface deformation and the convection, PIV technique has been used to measure the velocity of the flow field. The Particle Image Velocimetry (PIV) is a powerful tool recently for measuring the velocity field in the experiment of fluid mechanics, and it records the flow structure in a cross-section simultaneously. A system of Digital Particle Image Velocimetry (DPIV) of DANTEC measurement technology A/S with FlowMap PIV 2000 Processor was used to quantitatively measure the 2-D velocity field in a vertical cross-section of the liquid layer, as shown in Figure 5. An argon ion laser was applied to illuminate the flow field. Pulse lights were shaped by an electro-optical shutter, which was controlled by a center processor with CCD camera synchronization. The resolution of CCD camera is $768 \times 484$ pixels with $11.6 \times 13.6 \mu \mathrm{m}$ pixel pitch. The aluminum powder spheres of $10 \mu \mathrm{m}$ in diameter as tracer particles are suspended in the liquid layer. The density of the particles is $2.7 \mathrm{~g} / \mathrm{cm}^{3}$, which is close to the density of FC-70 liquid. By using a cycling lens, a $1.0 \mathrm{~mm}$ thin light sheet is shaped to illuminate a vertical cross-section at the center part of the cavity. This cross-correlation technique of DPIV image matching processing was used to obtain both magnitude and direction of velocity vector at the same time. 


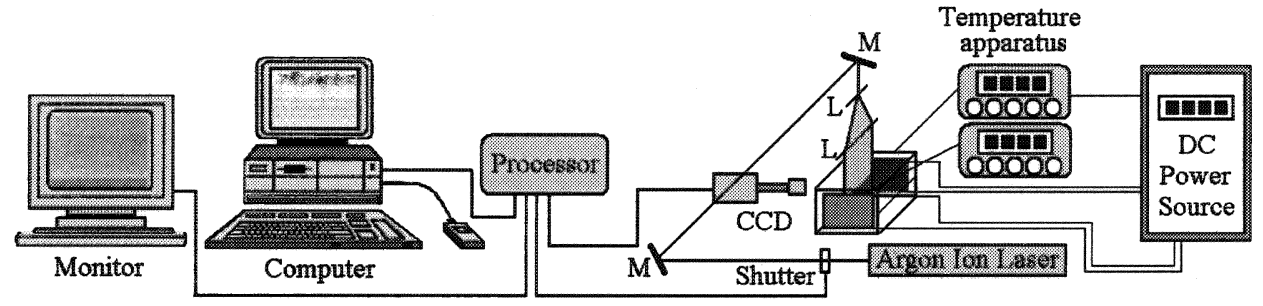

\section{Experimental Result}

Fig. 5 Arrangement of PIV in the experiment

In the present experiment, there is $4 \mathrm{~mm}$ in height of the silicon oil layer in the experimental cavity. The temperature difference between the two sides of the cavity increased from $0^{\circ} \mathrm{C}$ to $60^{\circ} \mathrm{C}$ with the increasing temperature rate of $1^{\circ} \mathrm{C} / \mathrm{Sec}$. The optical interference method measures the surface deformation and the surface wave of the convection. The interference fringes at the temperature difference of $0^{\circ} \mathrm{C}$ is modulated to be horizontal, which is to be as the original fringe for the latter calculation. The deformation of the interference fringes, which is to be as the metamorphic fringe, has been captured, which demonstrates that the surface deformation appears when the steady convection is developed. The interference fringes are shown as in Fig. 6, and the surface deformation and surface wave are calculated by Fourier transformation, shown as in Fig. 7. When the temperature difference is very small, which is less than $3^{\circ} \mathrm{C}$, the cooled side is higher than the heated side because of the liquid moving from the hot side to the cool side; with the temperature difference between the two sides increasing, the tilting surface is enhanced. The interference fringes in the present experiment are more and more declining in this process. When the temperature difference is larger than $21^{\circ} \mathrm{C}$, a wave crest appears at the hot side, and moves to the cool side gradually, and then appears at the hot side. The interference fringes curl at the hot side firstly, and then this curl fringes move to the cool side gradually. The surface deformation in different temperature and the wave amplitude of the surface wave have been calculated shown as in Fig. 7.

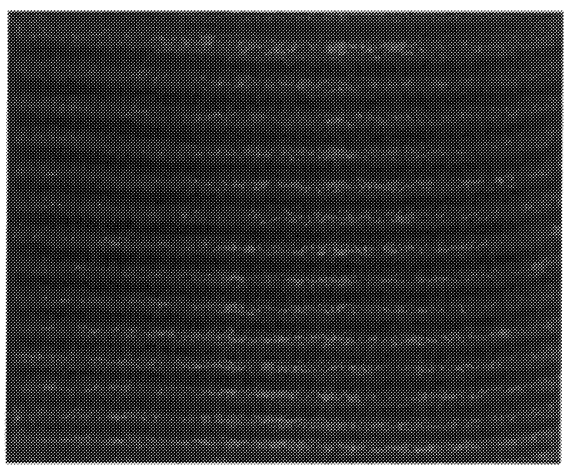

(a) $\Delta \mathrm{T}=0^{\circ} \mathrm{C}$ 


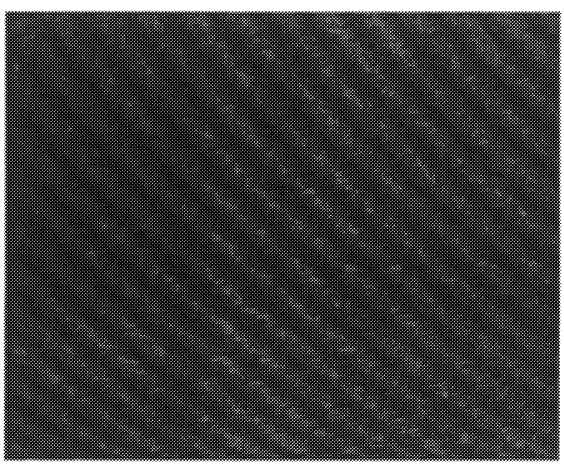

(b) $\Delta \mathrm{T}=14.8^{\circ} \mathrm{C}$

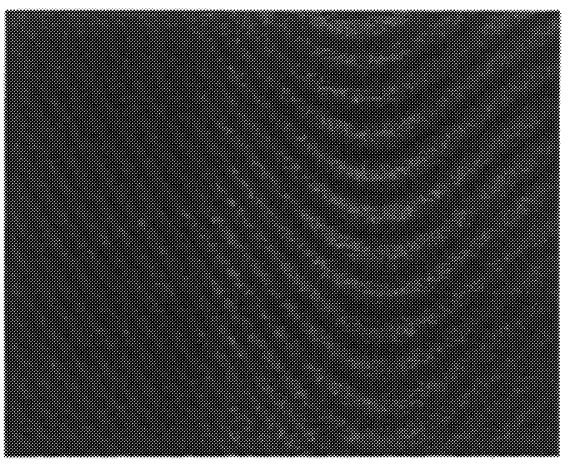

(d) $\Delta \mathrm{T}=49.4^{\circ} \mathrm{C}$

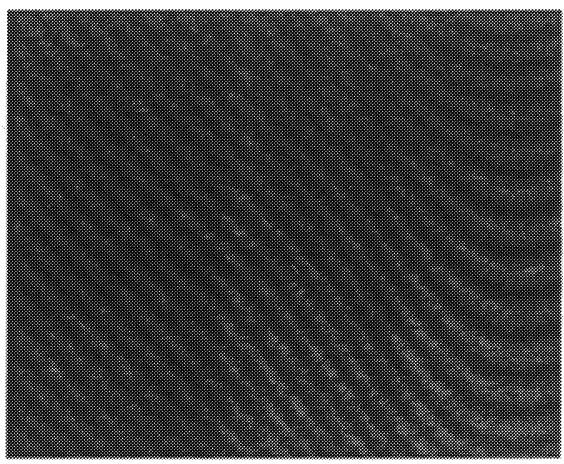

(c) $\Delta \mathrm{T}=34.2^{\circ} \mathrm{C}$

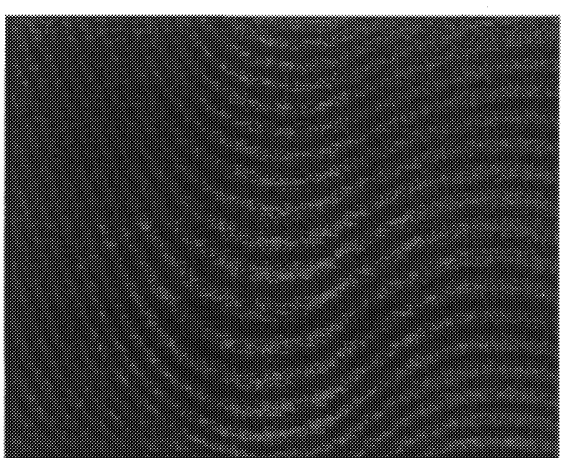

(e) $\Delta \mathrm{T}=56.0^{\circ} \mathrm{C}$

Fig. 6 The interference fringe
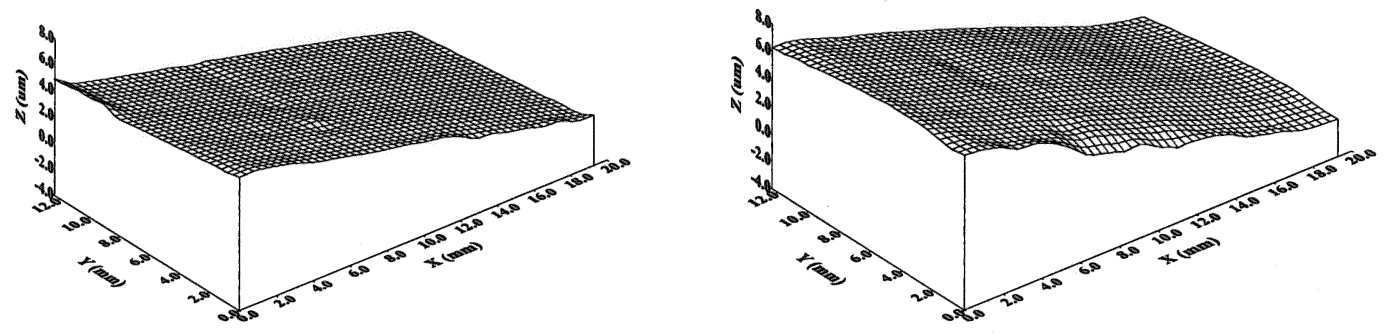

$\Delta \mathrm{T}=14.8^{\circ} \mathrm{C}$

$\Delta \mathrm{T}=34.2^{\circ} \mathrm{C}$
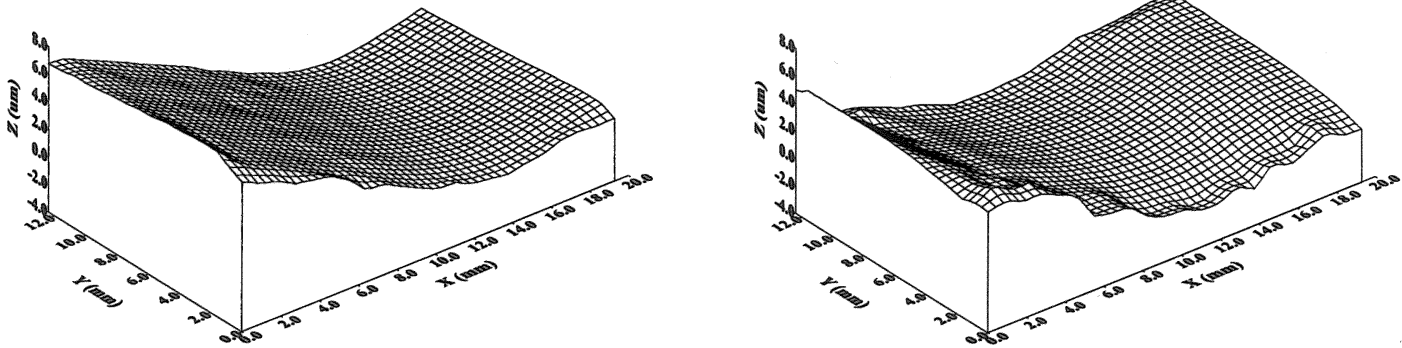

$\Delta \mathrm{T}=49.4^{\circ} \mathrm{C}$

$\Delta \mathrm{T}=56.0^{\circ} \mathrm{C}$

Fig. 7 The surface deformation and the surface wave 
The particle image velocimetry (PIV) technique observes the feature and the development of the thermal capillary convection. The measured area $6 \mathrm{~mm} \times 4 \mathrm{~mm}$ locates on the center of the liquid layer surface. When the temperature difference is small, the thermal capillary convection appears, which is the flow from the heated side to the cool side, and then back to the heated side. When the temperature difference is increased to $55^{\circ} \mathrm{C}$, the un-steady convection is appeared, the centers of eddies move from the heated side to the cool side. The velocity field is shown as in Fig. 8.

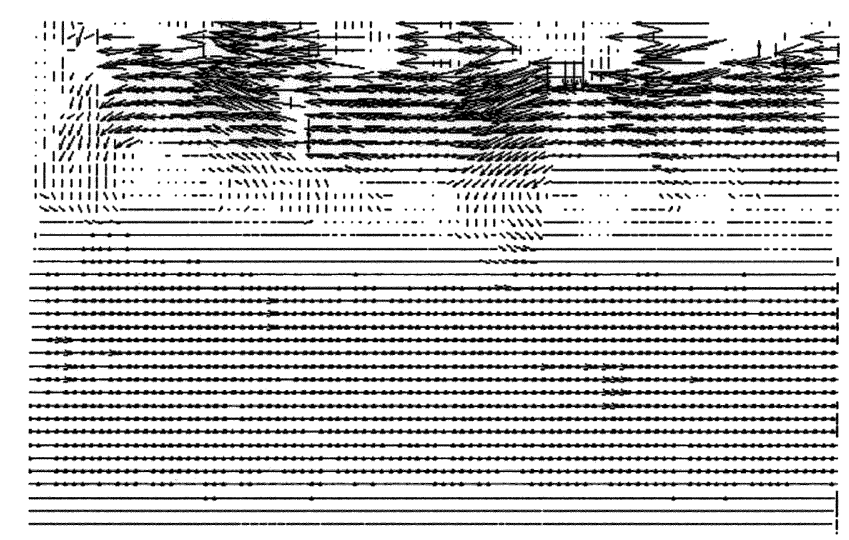

Fig. 8 The velocity field of the thermal capillary convection $(7 \mathrm{~mm} \times 4 \mathrm{~mm})$

\section{Conclusion}

An optical diagnostic system consisting of the Michelson interferometer with the image processor has been developed for the study of the kinetics of the thermal capillary convection. The PIV technique and this optical interferometer have been used to observe and measure the surface deformation and surface wave of the capillary convection in a rectangular cavity with different temperature's sidewalls. The particle image velocimetry (PIV) technique observes the feature and the development of the thermal capillary convection. The optical interference method measures the surface deformation and the surface wave of the convection. Fourier transformation is used to image processing. The quantitative results of the surface deformation and surface wave have been calculated from the interference fringe pattern. The present experiment proved that PIV technique and interferometer are valid optical means to study flow phenomena.

\section{References}

1. W. R. Hu, S. C. Xu, Microgravity Fluid Mechanics, Science press, China (in Chinese) 1999.

2. M. Hamed, J. M. Floryan, Marangoni convection. Part 1. A cavity with differentially heated sidewalls, J. Fluids, (2000), vol. 405, 79-110.

3. M. Takeda, K. Mutoh, Fourier transform profilometry for the automatic measurement 3-D object shapes. Appl. Opt., 1983, 22(24):3977-3982.

4. T. R. Judge \& P. J. Bryanston-Cross, A Review of Phase Unwrapping Techniques in Fringe Analysis, Opt. \& Lasers in Eng., 1994, 199-239. 